- Original-

\title{
Effect of the Surface Potential of the Hemodialysis Membrane and the Electrical Charge of the Gadolinium Contrast Medium on Dialyzability
}

\author{
Susumu Okada ${ }^{1}$, Kohei Inoue ${ }^{1}$, Tetsuji Kijima ${ }^{1}$, Kiwayo Katagiri ${ }^{2}$ and Tatsuo Kumazaki ${ }^{2}$ \\ ${ }^{1}$ Department of Radiology, Chiba-Hokuso Hospital, Nippon Medical School \\ ${ }^{2}$ Department of Radiology, Nippon Medical School
}

\begin{abstract}
Purpose: To search for methods of improving the excretion of injected gadolinium contrast medium (GdCM) in hemodialysis (HD) patients, we investigated the effect of the surface potential of HD membranes and the electrical charge of GdCM on the dialyzability of GdCM.

Materials and Methods: Ionic (Gd-DTPA) or non-ionic (Gd-DO 3 A) GdCM solutions were dialyzed using a clinical HD unit. Two types of HD membranes, AN 69 with a surface potential and PMMA without, were used. GdCM clearance was then calculated.

Results: Gd-DTPA clearance was significantly higher for PMMA membranes than for AN 69 membranes. Gd-DO 3 A clearance was slightly higher for AN 69 membranes than for PMMA membranes. The difference in Gd-DTPA and Gd-DO 3 A clearance values was not significant when PMMA membranes were used.

Conclusion: These data indicate that non-ionic GdCM is preferable to ionic GdCM in patients receiving dialysis through an electrically positive membrane. Either ionic or non-ionic $\mathrm{GdCM}$ can be used when a normal dialysis membrane is being used.
\end{abstract}

(J Nippon Med Sch 2003; 70: 12-15)

Key words: hemodialysis, gadolinium contrast medium, membrane potential

\section{Introduction}

In hemodialysis (HD) patients, HD is the only method available for excreting injected gadolinium contrast medium $(\mathrm{GdCM})$. We previously reported that $\mathrm{GdCM}$ can be used in HD patients ${ }^{1}$; however, the amount of GdCM excreted during a single HD session is less than $80 \%$ of the injected amount ${ }^{1.2}$. In normal subjects, about $90 \%$ of the injected GdCM is excreted within the first 24 hours $^{3}$; thus, the halftime of the injected GdCM is longer in $\mathrm{HD}$ patients than in normal subjects. Consequently, the selection of an appropriate $\mathrm{GdCM}$ and $\mathrm{HD}$ method is important. Unlike iodine contrast medium, in which the ionic form is now rarely used in intra-vascular injections, both ionic and non-ionic GdCM are used during MR examinations. To investigate the difference in hemodialyzability of non-ionic and ionic $\mathrm{GdCM}$, we studied the relationship between the electrical charge of the contrast media and the surface potential of the HD membrane.

Correspondence to Susumu Okada, Department of Radiology, Chiba-Hokuso Hospital, Nippon Medical School, 1715 Kamagari, Inba-mura, Inba-gun, Chiba 270-1613, Japan

E-mail: okada@nms.ac.jp

Journal Website (http://www.nms.ac.jp/jnms/) 


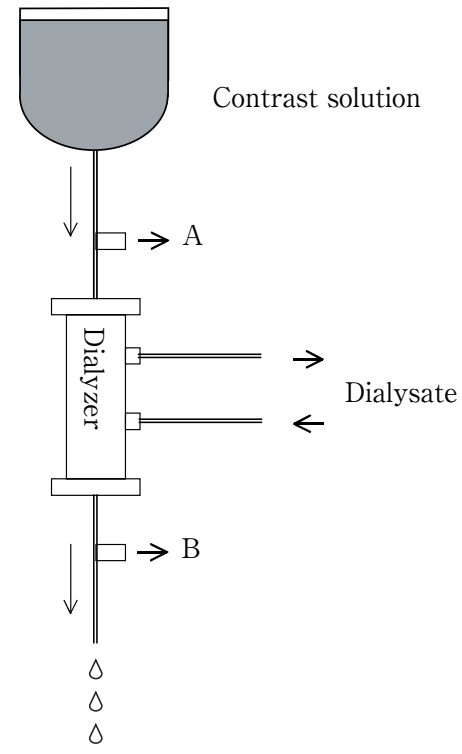

Fig. 1 Schematic diagram of the experiment model.

\section{Materials and Methods}

Following the reports of Ueda et $\mathrm{al}^{4}{ }^{4}$ and Furukawa et al., GdCM solution was dialyzed without reperfusion (Fig. 1). Fifteen milliliters of GdCM was diluted in $5 \mathrm{~L}$ of natural saline. Either ionic Gd-DTPA (Magnevist, Schering, Japan) or nonionic Gd-DO 3 A (Prohance, Eizai, Japan) was used. The GdCM solution flow and the dialysate flow were $200 \mathrm{ml} / \mathrm{min}$ and $500 \mathrm{ml} / \mathrm{min}$, respectively. All experiments were conducted at $36^{\circ} \mathrm{C}$. An $\mathrm{AN} 69$ membrane (Hospal, Japan) with a negative surface potential $(-100 \mathrm{mv})$ and a PMMA membrane

(Filtrizer BK, Torey, Japan) with no surface potential were used for the dialysis. The surface area of each membrane was $1.3 \mathrm{~m}^{2}$; the pore size was about $45 \AA$ for $\mathrm{AN} 69$ and $40 \AA$ for PMMA, and the membrane thickness was $45 \mu$ for AN 69 and $30 \mu$ for PMMA. Five milliliters of the samples were obtained at the afferent (A) and the efferent (B) ends of the dialyzer, and each measurement was repeated ten times for each GdCM and each HD membrane. The gadolinium concentration of each sample was determined using inductively coupled plasma spectrometry (SPS 1200 A; Seiko, Japan). GdCM clearance was calculated using the following equation :
Clearance $=\frac{(\mathrm{Ca}-\mathrm{Cv})}{\mathrm{Ca} \times \mathrm{Qb}}$

Ca: afferent concentration

Cv: efferent concentration

Qb: perfusion rate $(200 \mathrm{~mL} / \mathrm{min})$

The results were analyzed using the Student $t$ test with SAS Release 6.12 software package for Windows 98 .

\section{Results}

The average clearance $(\mathrm{ml} / \mathrm{min})$ was 115.5 for GdDO 3 A/AN 69 (GdCM/HD membrane), 108.1 for Gd-DO 3 A/PMMA, 90.0 for Gd-DTPA/AN 69, and 106.0 for Gd-DTPA/PMMA (Fig. 2).

When these combinations were analyzed according to the type of GdCM used, the average clearance value for Gd-DO $3 \mathrm{~A} / \mathrm{AN} 69$ was higher than that for Gd-DO 3 A/PMMA, but the difference was not significant $(p=0.0221)$. The average clearance value for Gd-DTPA/AN 69 was significantly lower than that for Gd-DTPA/PMMA $(p<0.01)$. When analyzed according to the type of membrane used, the average clearance value for GdDO $3 \mathrm{~A} / \mathrm{AN} 69$ was significantly higher than that for Gd-DTPA/AN $69(\mathrm{p}<0.01)$; the average clearance values for Gd-DO $3 \mathrm{~A} / \mathrm{PMMA}$ and GdDTPA/PMMA were not significantly different.

\section{Discussion}

Three to four HD sessions are usually necessary for the complete excretion of GdCM in $\mathrm{HD}$ patients 1,2,6. Thus, injected GdCM can remain within a patient's body for up to one week when the usual HD method is employed. No side effects of GdCM have been reported in previous clinical studies ${ }^{1,2}$; nevertheless, an improved HD method is desirable to reduce the possibility of unknown side effects. Prompt excretion of GdCM might be especially important in acute renal failure patients. Recently, high flux membranes have been reported to exhibit better dialyzability than low flux membranes for the excretion of $\mathrm{GdCM}^{4}$ and iodinated contrast media ${ }^{7.8}$. Ueda et al. $^{4}$ reported that molecular weight was the main factor affecting the clearance of contrast media 


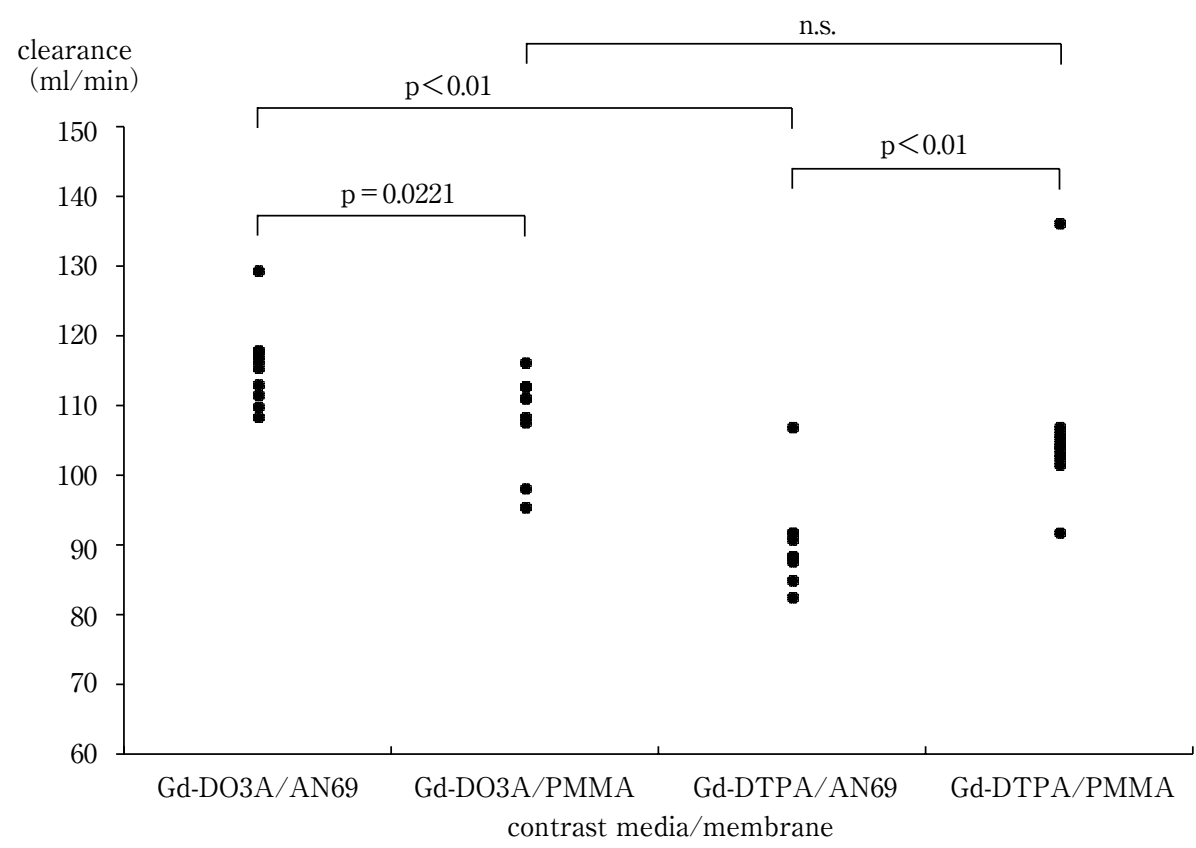

Fig. 2 Graph indicates clearance data in each combination of GdCM and HD membrane. GdDTPA clearance was significantly higher for PMMA membranes than for AN 69 membranes. Gd-DO 3 A clearance for AN 69 membranes was slightly higher than for PMMA membranes. The difference in Gd-DTPA and Gd-DO 3 A clearance values was not significant when PMMA membranes were used.

and that permeability was significantly higher when membranes with a large pore size were used. However, Matzkies et al. $^{9}$ reported that the elimination of iodinated contrast media was not dependent on the pore size of the membrane and concluded that the efficiency of contrast media elimination depended upon the HD procedure. Many factors, including pore size, hydrophilic property, thickness, surface area, distribution of pores, surface potential and absorption ability, may affect the permeability of membranes. Furukawa et al. ${ }^{5}$ showed that the electrical charge and the molecular aggregation of iodinated contrast medium were not important with regard to dialyzability. However, there has been no report that focused on the role of the membrane surface potential. Under such backgrounds, we performed this study and investigated the difference between ionic- and nonionic contrast medium.

AN 69 is a high-performance membrane with a negative surface potential that is close to that of cellular basement membranes. Using AN 69, a significant difference in GdCM clearance was observed between ionic and non-ionic GdCM. The clearance of Gd-DTPA was significantly higher for PMMA membranes than for AN 69. The clearance of Gd-DO 3 A was slightly higher for AN 69 membranes than for PMMA. No difference in GdDTPA and Gd-DO 3 A clearance values were observed for PMMA membranes. These data indicate that the membrane surface potential affects the elimination of ionic GdCM. The slightly higher clearance value for non-ionic GdCM that was obtained using the AN 69 membrane is probably related to some factor other than membrane potential. The similarity in clearance values for ionic and non-ionic contrast media when a non-positive membrane is used agree with the reports by Furukawa et al. ${ }^{5}$ and Choyke et al. ${ }^{6}$.

In conclusion, non-ionic GdCM is more easily excreted than ionic GdCM in patients undergoing hemodialysis with an electrically positive membrane. Both ionic and non-ionic GdCM can be used when a usual membrane is employed. Radiologists should consult the HD department with regard to the type of GdCM that should be used when contrastenhanced MRI examinations are performed in HD patients. 


\section{Acknowledgement}

The authors wish to thank Mr. Eiji Kushima, Miss Yukie Taguchi, Mr. Kouichirou Misono and Mr. Yasuo Katayama for their assistance with the experiments.

\section{References}

1. Okada S, Katagiri K, Kumazaki T and Yokoyama H : Safety of gadolinium contrast agent in hemodialysis patients. Acta Radiol 2001; 42: 339-526.

2. Krahe VT, Götz R, Lackner K, Haustein J, Klemenz $\mathrm{U}$, Landdwehr $\mathrm{P}$, Heidbreder $\mathrm{E}$ and SchuhmannGiampieri G : Pharmakokinetik von Gadolinium- DTPA bei dialysepflichtiger chronisher Niereninsuffizienz. RÖFO 1992; 156: 523-341.

3. Weinmann HJ, Brasch RC, Press WR, Wesbey GE: Characteristics of gadolinium-DTPA complex: a potential NMR contrast agent. AJR 1984;142: 619-624.

4. Ueda J, Furukawa T, Higashino K. Yamamoto T. Ujita H. Sakaguchi K and Araki Y:Permeability of iodinated and MR contrast media through two types of hemodialysis membrane. Europ Radiol 1999; 31: 76-80.

5. Furukawa T, Ueda J, Higashino K, Takahashi S, Sakaguchi $\mathrm{K}$ and Imai $\mathrm{T}$ :Permeability of contrast media through hemodialysis membrane. Acta Radiol 1997; 38: 918-921.

6. Coyke PL, Girton ME, Vaughan EM, Frank JA and Austin III HA : Clearance of gadolinium chelates by hemodialysis: An in vitro study. JMRI 1995; 4 : 470-472.

7. Berg KJ, Rolfsen B and Stake G: Iodixanol is readily eliminated by hemodialysis. Acta Radiol 1998; 39: 372-374.

8. Schindler R, Stahl C, Venz S, ludat K, Krause W and Frei U:Removal of contrast media by different extracorporeal treatments. Nephrol Dial Transplant 2001; 16: 1471-1474.

9. Matzkies FK, Tmbach B, Kisters K, Schuhmann G, Hohage $\mathrm{H}$ and Schaefer RM : Clearance of iopromide during haemodialysis with high- and low-flux membranes. Acta Radiol 1999; 40: 220-223.

(Received, July 4, 2002)

(Accepted, August 12, 2002) 